\title{
Inducers of Aflatoxin Biosynthesis from Colonized Maize Kernels Are Generated by an Amylase Activity from Aspergillus flavus
}

\author{
C. P. Woloshuk, J. R. Cavaletto, and T. E. Cleveland
}

First and second authors: Department of Botany and Plant Pathology, Purdue University, West Lafayette, IN 47907; and third author: U.S. Department of Agriculture/Agricultural Research Service, Southern Regional Research Center, New Orleans, LA 70179. Accepted for publication 8 October 1996.

\begin{abstract}
Woloshuk, C. P., Cavaletto, J. R., and Cleveland, T. E. 1997. Inducers of aflatoxin biosynthesis from colonized maize kernels are generated by an amylase activity from Aspergillus flavus. Phytopathology 87:164-169.

Aflatoxin biosynthesis was induced by compounds in filtrates (EF) obtained from cultures consisting of ground maize kernels colonized by Aspergillus flavus. The inducing activity increased to a maximum at 4 days of incubation and then decreased. Amylase activity was detected in the EF, suggesting that the inducers are products of starch degradation (glucose, maltose, and maltotriose). Analysis of the enzyme by isoelectric focusing electrophoresis indicated a single $\alpha$-amylase with a pI of 4.3. No maltase or amyloglucosidase was detected in the EF. High-pres-
\end{abstract}

ABSTRACT sure liquid chromatography analysis of the EF indicated the presence of glucose, maltose, and maltotriose in near-equal molar concentrations (about $15 \mathrm{mM}$ ). With a $\beta$-glucuronidase (GUS) reporter assay consisting of A. flavus transformed with an aflatoxin gene promoter-GUS reporter gene fusion to monitor induction of aflatoxin biosynthesis, the minimum concentration of glucose, maltose, or maltotriose that induced measurable GUS activity was determined to be $1 \mathrm{mM}$. These results support the hypothesis that the best inducers of aflatoxin biosynthesis are carbon sources readily metabolized via glycolysis. They also suggest that $\alpha$ amylase produced by A. flavus has a role in the induction of aflatoxin biosynthesis in infected maize kernels.
Aspergillus flavus Link:Fr. causes an ear and kernel rot of maize (Zea mays L.). Although A. flavus is a common fungus growing as a saprophyte on dead plant debris, infection of living tissue can occur when plants are damaged by insect pests or severely stressed by drought and high temperatures. Such conditions are most common in the southeastern and southwestern United States $(14,25)$. This disease, known as Aspergillus ear rot, is of economic concern, because aflatoxins are produced by A. flavus. Aflatoxins are a family of structurally related secondary metabolites that, when ingested by a number of animal species, are potent liver toxins and carcinogens. The U.S. Food and Drug Administration has set legal limits $(20 \mathrm{ppb})$ on the amount of aflatoxin that can be present in grain sold through interstate commerce, and many international markets have established limits that are often much lower than those set by the United States.

Control of Aspergillus ear rot is difficult. Other than irrigation, there is no effective management practice for controlling the disease (25). Although genetic resistance has potential, no commercial hybrids with resistance are currently available $(9,10,17,30$, 33). Several researchers have identified maize genotypes with varying degrees of resistance; however, defining the specific loci involved has been arduous. A major difficulty has been the lack of uniform environmental conditions from year to year, making field selection of resistance difficult $(14,25)$.

One approach for identifying host factors involved in resistance and susceptibility to infection by A. flavus and aflatoxin biosynthesis is the careful examination of the host-fungus interaction. For this purpose, we developed a reporter-gene assay that can

Corresponding author: C. P. Woloshuk

E-mail address: woloshuk@btny.purdue.edu

Publication no. P-1996-1218-02R

This article is in the public domain and not copyrightable. It may be freely reprinted with customary crediting of the source. The American Phytopathological Society, 1997. readily measure molecular activation of aflatoxin biosynthesis (15). A DNA construct was made with the promoter of one pathway gene (verl) cloned immediately upstream of the $\beta$-glucuronidase (GUS) gene (uidA) from Escherichia coli. The product of the verl gene is involved in the conversion of versicolorin A to demethylsterigmatocystin in the aflatoxin biosynthetic pathway $(11,31)$. Analysis of RNA transcript analysis, aflatoxin accumulation, and GUS expression showed that the verl:GUS construct in an A. flavus transformant (GAP13-22) reliably monitors the promoter activity of the verl gene (15). These data also demonstrated that the promoter-GUS construct can be utilized effectively to examine the timing and magnitude of gene expression of at least one gene in the pathway (15).

With the GUS reporter, an aflatoxin-inducing activity was detected previously in culture filtrates of the aflatoxigenic A. flavus strain NRRL 3357 grown on maize kernels (15). The inducing activity passed through ultrafiltration membranes with $10-\mathrm{kDa}$ exclusion and was not inactivated by $15 \mathrm{~min}$ of autoclaving. Filtrates from suspensions of ground maize kernels without the fungus also had inducing activity. However, the activity was variable between experiments, and it was completely lost after filtration through glass microfilters (15). These data suggested that the inducing activity was the degradation products of a complex seed molecule. A major component of the ground maize kernels was starch. Therefore, the objective of the this study was to explore the possibility that an amylase produced by A. flavus and the degradation products of starch have a role in the aflatoxin induction mechanism in colonized maize kernels.

\section{MATERIALS AND METHODS}

Fungal isolates and culture methods. A. flavus strain GAP1322 is a transformant of strain 656-2 (whi, leu, aflR) containing the verl promoter:GUS-reporter construct GAP13 and the AflR construct B9X2 $(15,26,36)$. Strain NRRL 3357 was obtained from U.S. Department of Agriculture Northern Regional Research 
Center in Peoria, IL. Fungi were grown and maintained on potato dextrose agar.

For measuring induction of aflatoxin biosynthesis, $1 \mathrm{ml}$ of peptone mineral salts (PMS) medium (8) and $10^{6}$ conidia of transformant GAP13-22 were placed in each well of a 24-well tissue culture plate. After 3 days of incubation at $28^{\circ} \mathrm{C}$, the PMS medium was replaced with known sugar solutions or filtrates from $A$. flavus cultures containing putative-inducing activities. Previous studies indicate that the specific activity of GUS can vary between experiments, but within an experiment there is little variation between replicates (15). Therefore, each experiment included a positive (aflatoxin-inducing) control: PMS plus $60 \mathrm{~g} /$ liter of glucose (PMSG) as a replacement medium (8). Twenty-four hours after the media replacement, mycelia were removed from the wells and stored at $-20^{\circ} \mathrm{C}$ until GUS analyses were performed. Potential aflatoxin-inducing sugars were tested as aqueous solutions containing $10 \mathrm{mM}$ leucine. Maize kernel-derived culture filtrates (EF) were produced by growing A. flavus strain NRRL 3357 on ground maize kernels. Kernels (30 g) of Pioneer hybrid 3369A were ground to a fine powder, suspended in $40 \mathrm{ml}$ of water, autoclaved for $20 \mathrm{~min}$, and inoculated with $10^{7}$ conida. Filtrates of cultures grown on amylopectin (AP) were produced by growing strain NRRL 3557 on media containing $35 \mathrm{mM}$ sodium nitrate, $4.4 \mathrm{mM}$
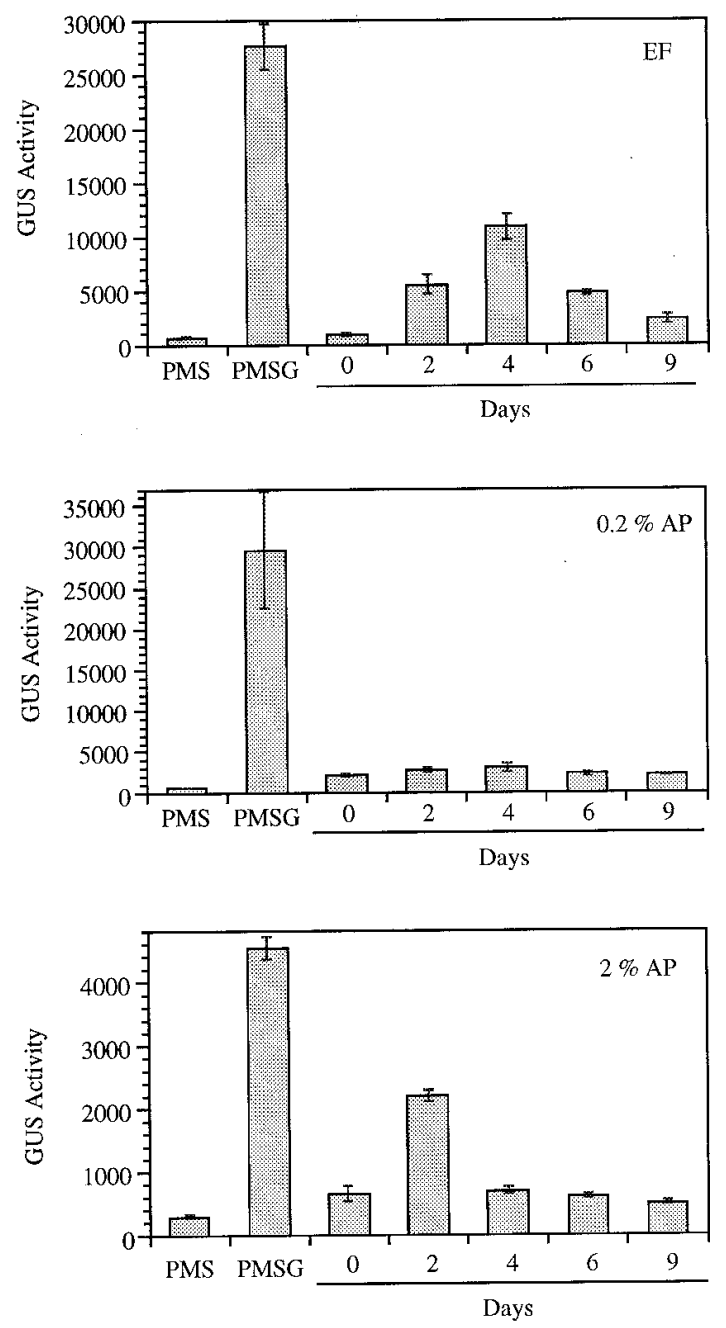

Fig. 1. Accumulation of $\beta$-glucuronidase (GUS)-inducing activity in maize (EF), 0.2 and $2 \%$ amylopectin (AP) culture filtrates. Aspergillus flavus transformant GAP13-22 was grown in peptone mineral salts (PMS) medium for 3 days at $28^{\circ} \mathrm{C}$. At zero time the culture medium in the bioassay was replaced. GUS activity was determined after $24 \mathrm{~h}$ of incubation. Values are the average of three replicates and the bars represent the standard errors. GUS values are umoles 4-methylumbelliferyl $/ \mathrm{min} / \mathrm{mg}$ of protein. PMS and PMS plus 60 $\mathrm{g} /$ liter of glucose are the negative and positive controls, respectively. dipotassium phosphate, $2.0 \mathrm{mM}$ magnesium sulfate, $6.7 \mathrm{mM}$ potassium chloride, $36 \mu \mathrm{M}$ ferrous sulfate, and 0.2 or $2 \%$ amylopectin (corn starch). Cultures were filtered through Miracloth (Calbiochem-Behring, La Jolla, CA), and the filtrates were centrifuged to remove fungal and insoluble material. Prior to adding the culture filtrates to the GUS bioassay, leucine was added at a final concentration of $10 \mathrm{mM}$. All induction experiments were repeated at least twice.

GUS analysis. Frozen mycelium was placed in a $1.5-\mathrm{ml} \mathrm{mi-}$ crofuge tube with $20 \mathrm{mg}$ of glass beads (100 to $200 \mu \mathrm{m}$ ) and 300 $\mu \mathrm{l}$ of GUS extraction buffer (50 mM sodium phosphate [pH 7.0], $10 \mu \mathrm{M} \beta$-mercaptoethanol, $10 \mathrm{mM}$ sodium EDTA, 0.1\% Triton X100 , and $0.1 \%$ sodium lauryl sarcosine). Mycelium was homogenized for $30 \mathrm{~s}$ using a pestle. After centrifugation for $2 \mathrm{~min}$ at $14,000 \times g$, the supernatant was assayed for protein and GUS activity. Protein was measured by the method of Bradford (4) with bovine serum albumin as the standard. GUS was determined by the addition of $50 \mu \mathrm{l}$ of fungal extract to $500 \mu \mathrm{l}$ of assay buffer (1.25 mM 4-methylumbelliferyl [MU] $\beta$-D-glucuronide in extraction buffer) preincubated at $37^{\circ} \mathrm{C}$. After 0,5 , and $10 \mathrm{~min}, 100 \mu \mathrm{l}$ of buffer was removed from reaction tubes and transferred to microfuge tubes containing $900 \mu \mathrm{l}$ of $0.2 \mathrm{M} \mathrm{Na}_{2} \mathrm{CO}_{3}$ to stop the reaction (15). The enzyme product MU was measured with a Hoefer TKO100 fluorometer (Hoefer Scientific Instruments, San Francisco).

High-pressure liquid chromatography (HPLC) analysis. Sugars were separated with a Carbo-Pac PA1 anion-exchange column (Dionex Corp., Sunnyvale, CA) and a Dionex BioLC (Dionex Corp.) equipped with a pulsed amperometric detector. The sugars were eluted using a 20-min linear gradient from $200 \mathrm{mM}$ $\mathrm{NaOH}$ to $200 \mathrm{mM} \mathrm{NaOAc}$ in $200 \mathrm{mM} \mathrm{NaOH}$, followed by a 20min linear gradient up to $500 \mathrm{mM} \mathrm{NaOH}$ in $500 \mathrm{mM} \mathrm{NaOAc}$. Prior to injection onto the column, culture filtrates were mixed 1:1 with ethanol and placed on ice for $30 \mathrm{~min}$. After centrifugation, the supernatants were dried under vacuum and dissolved in water. Glucose, maltose, and maltotriose were used as standards.

Amylase analysis. The isoelectric focusing (IEF) procedures were as previously described $(12,28)$, except premanufactured IEF gels (Pharmacia LKB Biotechnology Inc., Piscataway, NJ) were used. These gels contained pharmalytes yielding a $\mathrm{pH}$ gradient of 3.5 to 9.5 . The $\mathrm{pH}$ gradient was determined directly with a flat $\mathrm{pH}$ electrode. Fifteen microliters of the culture filtrates were loaded on the IEF gel. Gels were analyzed for amylase activity with overlays containing $0.5 \%$ amylose in $1 \%$ agarose. Amylase was

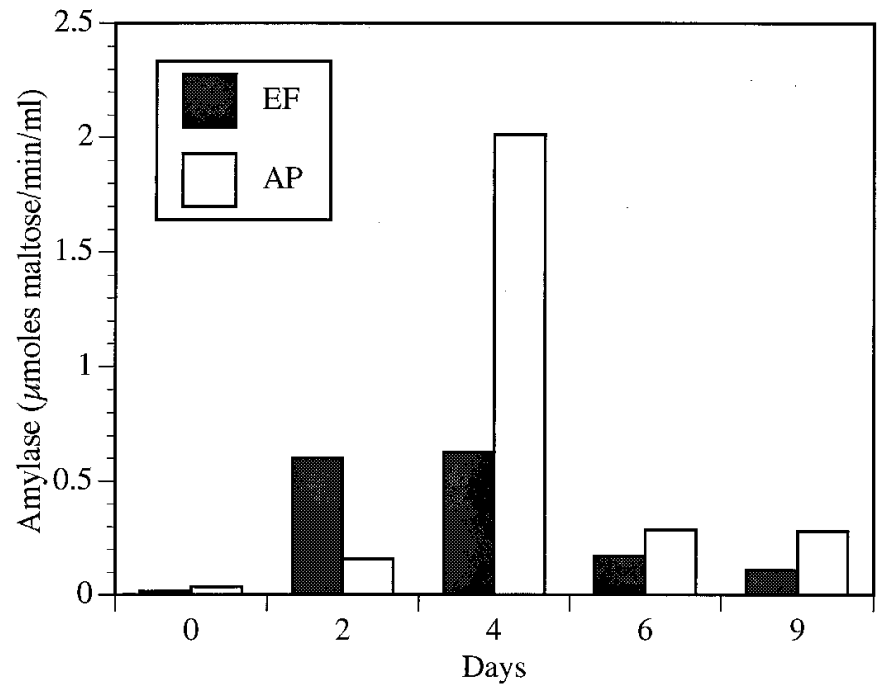

Fig. 2. Production of amylase in maize (EF) and $0.2 \%$ amylopectin (AP) culture filtrates. Aspergillus flavus NRRL 3357 was grown on maize kernels or AP. Amylase in the culture filtrates was measured at various time periods from 0 to 9 days. 
detected as a cleared zone after flooding overlays with an iodine solution $(0.05 \% \mathrm{wt} / \mathrm{vol})$.

Amylase activity was determined by incubating $0.1 \mathrm{ml}$ of the culture filtrates with $0.5 \mathrm{ml}$ of $3 \%$ soluble starch and $0.9 \mathrm{ml}$ of $100 \mathrm{mM}$ sodium acetate, $\mathrm{pH}$ 5.2. After an incubation period ranging from 3 to $30 \mathrm{~min}$ at $40^{\circ} \mathrm{C}$, reducing sugars were measured by the method of Nelson (24). Products were analyzed by incubating culture filtrates $(0.1 \mathrm{ml})$ with $0.1 \mathrm{ml}$ of $0.6 \%$ maltose, $0.6 \%$ maltotriose, $0.6 \%$ maltotetraose, or $0.6 \%$ maltoheptaose for $8 \mathrm{~h}$ at $40^{\circ} \mathrm{C}$. The enzyme reactions were stopped by heating at $100^{\circ} \mathrm{C}$ for $5 \mathrm{~min}$, and products were separated by thin-layer chromatography (TLC). Plates (silica gel G 60) were developed in $n$-propanol/water/ammonium hydroxide (70:30:1), and the products were detected by spraying the TLC plates with $30 \%$ sulfuric acid solution and charring at $100^{\circ} \mathrm{C}$.

\section{RESULTS}

Analysis of culture filtrates. A. flavus strain NRRL 3357 was grown on ground maize kernels or $0.2 \%$ AP for 9 days to produce
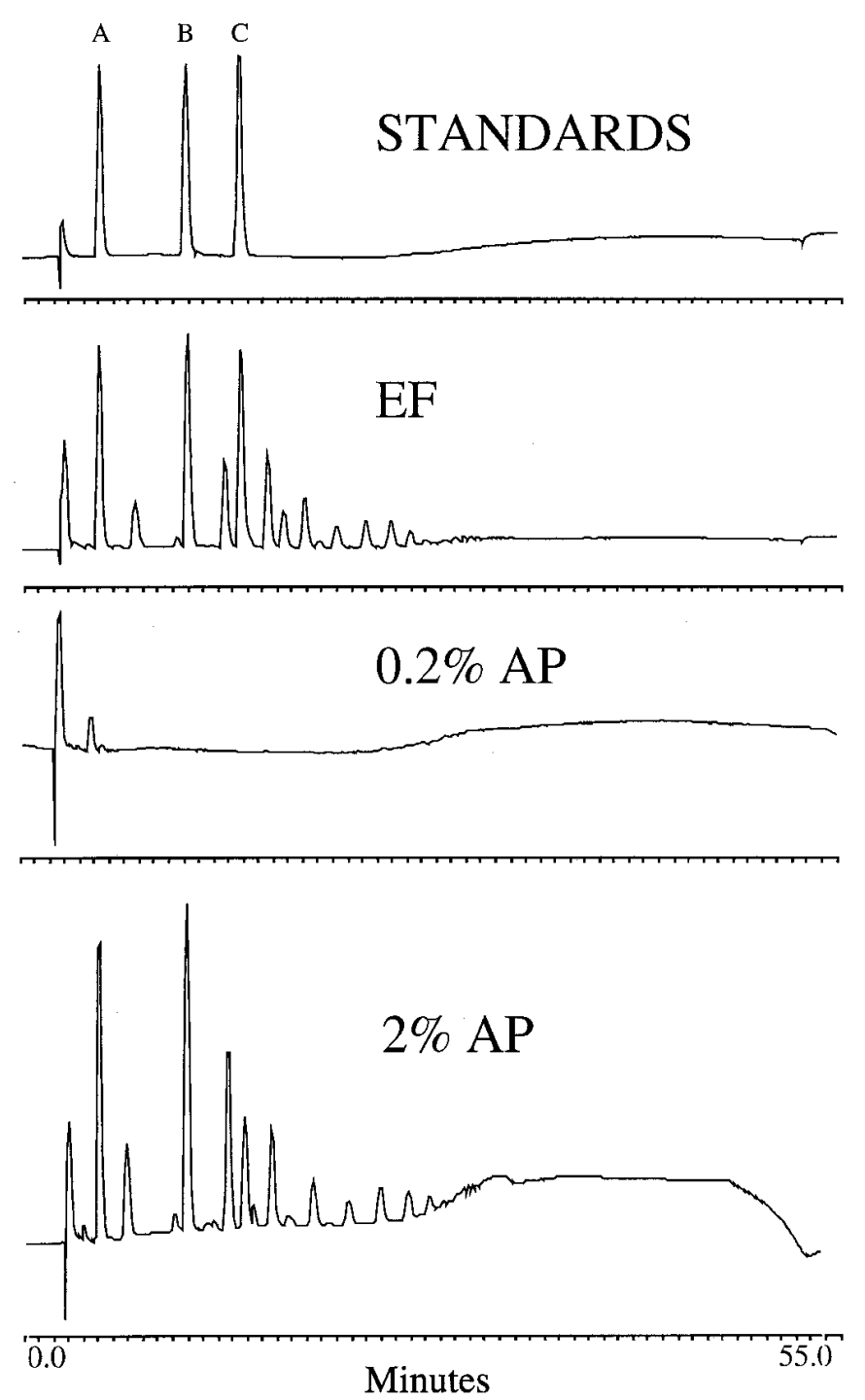

Fig. 3. Analysis of sugars in maize (EF), 0.2 and $2 \%$ amylopectin (AP) culture filtrates. Samples were loaded onto a Carbo-Pac PA1 anion-exchange column of a Dionex BioLC equipped with a pulsed amperometric detector. Sugars were eluted with a 20 -min linear gradient from $200 \mathrm{mM} \mathrm{NaOH}$ to 200 $\mathrm{mM} \mathrm{NaOAc}$ in $200 \mathrm{mM} \mathrm{NaOH}$, followed by a 20 -min linear gradient up to $500 \mathrm{mM} \mathrm{NaOH}$ in $500 \mathrm{mM} \mathrm{NaOAc}$. Standards were A, glucose; B, maltose; and $\mathbf{C}$, maltotriose. The EF culture filtrate was diluted 1,000-fold prior to injection, and $0.2 \%$ AP culture filtrate was diluted 10 -fold. The $2 \%$ AP culture filtrate was diluted 50 -fold prior to injection. test filtrates EF and AP, respectively. The culture filtrates were harvested at various times and tested for inducers of aflatoxin biosynthesis by the GUS-expression bioassay. As shown in Figure 1 , an inducing activity was detectable in the maize culture filtrates (EF) after 2 days of incubation. This inducing activity increased to a maximum by day 4 and then decreased. In contrast, no inducing activity was detected in the AP culture filtrates. The culture filtrates were also analyzed for amylase activity. Amylase activity in EF essentially paralleled that of the aflatoxin-inducing activity (Fig. 2). An increase in amylase activity was detected in 2-day-old cultures, reaching a maximum at 4 days, with decreased levels at days 6 and 9. Amylase in the AP culture filtrates followed a simi-

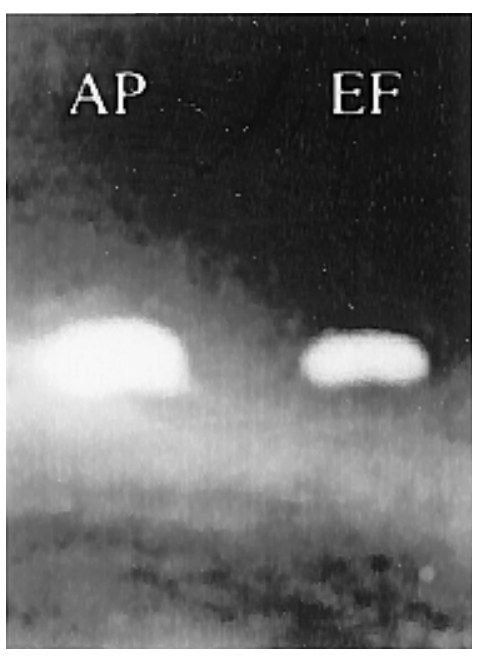

Fig. 4. Isoelectric focusing of the maize $\mathrm{EF}$ and $2 \%$ amylopectin (AP) culture filtrates. EF and $0.2 \% \mathrm{AP}$ culture filtrate $(15 \mu \mathrm{l})$ collected after 4 days of incubation were analyzed. Gel was overlayed with $0.5 \%$ amylase in $1 \%$ agarose. Amylase was detected as a cleared zone after flooding overlays with an iodine solution.
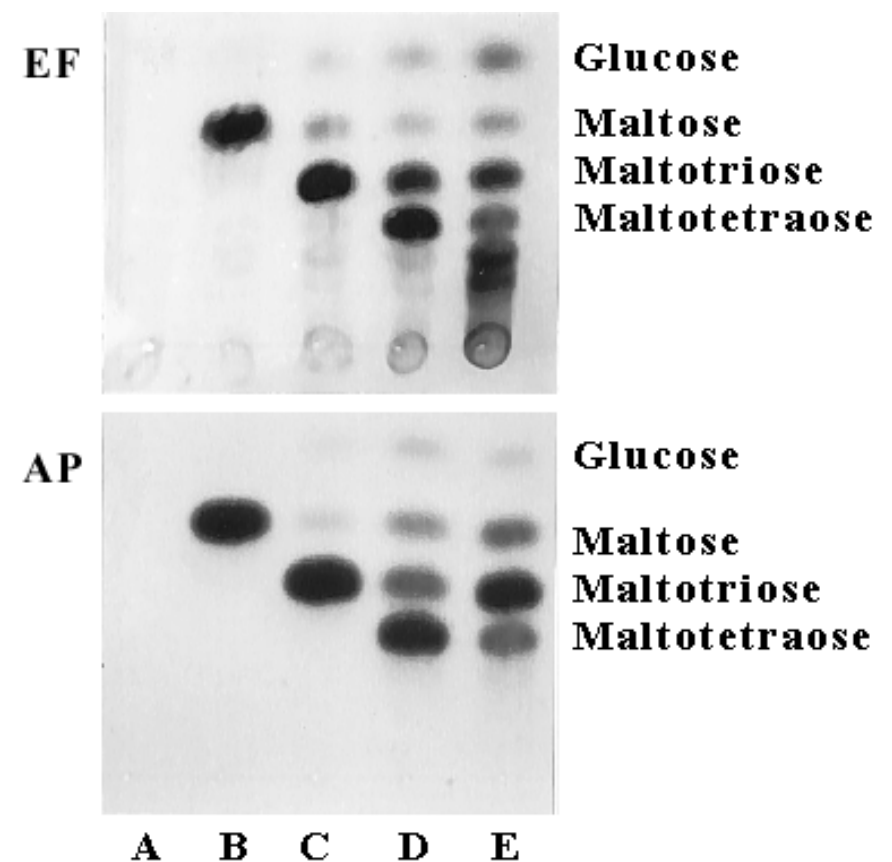

\section{Glucose}

Maltose

Maltotriose

Maltotetraose

Fig. 5. Thin-layer chromatographic (TLC) analysis of maltooligosaccharides digested by maize EF and $2 \%$ amylopectin (AP) culture filtrates. Culture filtrates were incubated for $8 \mathrm{~h}$ at $40^{\circ} \mathrm{C}$ with $\mathbf{A}$, no substrate; $\mathbf{B}, 0.6 \%$ maltose; C, $0.6 \%$ maltotriose; $\mathbf{D ,} 0.6 \%$ maltotetraose; or E, $0.6 \%$ maltoheptaose. After chromatographic development, products were detected by spraying the TLC plates with $30 \%$ sulfuric acid solution and charring at $100^{\circ} \mathrm{C}$. 
lar pattern as in EF. When the initial concentration of amylopectin in the cultures was increased from 0.2 to $2 \%$, an inducing activity was detected in the culture filtrates collected after 2 days of growth (Fig. 1). Low inducing activity was observed in filtrates collected at 4,6 , and 9 days.

The culture filtrates collected after 4 days of incubation were analyzed for sugars by HPLC. In the EF culture filtrates, carbohydrates eluted from the HPLC column at the same retention time as glucose, maltose, and maltotriose (Fig. 3). These sugars were present in near-equal molar concentrations (about $15 \mathrm{mM}$ ). In contrast, sugars were not detectable in $0.2 \%$ AP culture filtrates at any time point. Analysis of the $2 \%$ AP culture filtrates collected at 2 days indicated the presence of $4.5 \mathrm{mM}$ glucose, $7.5 \mathrm{mM}$ maltose, and $2.5 \mathrm{mM}$ maltotriose, as well as other sugar oligomers (Fig. 3). Trace amounts of these sugars were detected in filtrates from days 4, 6, and 9 (data not shown). Proteins in the 4-day EF and $0.2 \%$ AP culture filtrates were analyzed by isoelectric focusing. An overlay of the gels with AP and the subsequent staining with iodine indicated that both AP and EF contained a single band of amylase activity with a pI of 4.3 (Fig. 4). The culture filtrates were also incubated with maltooligosaccharides, and the products analyzed
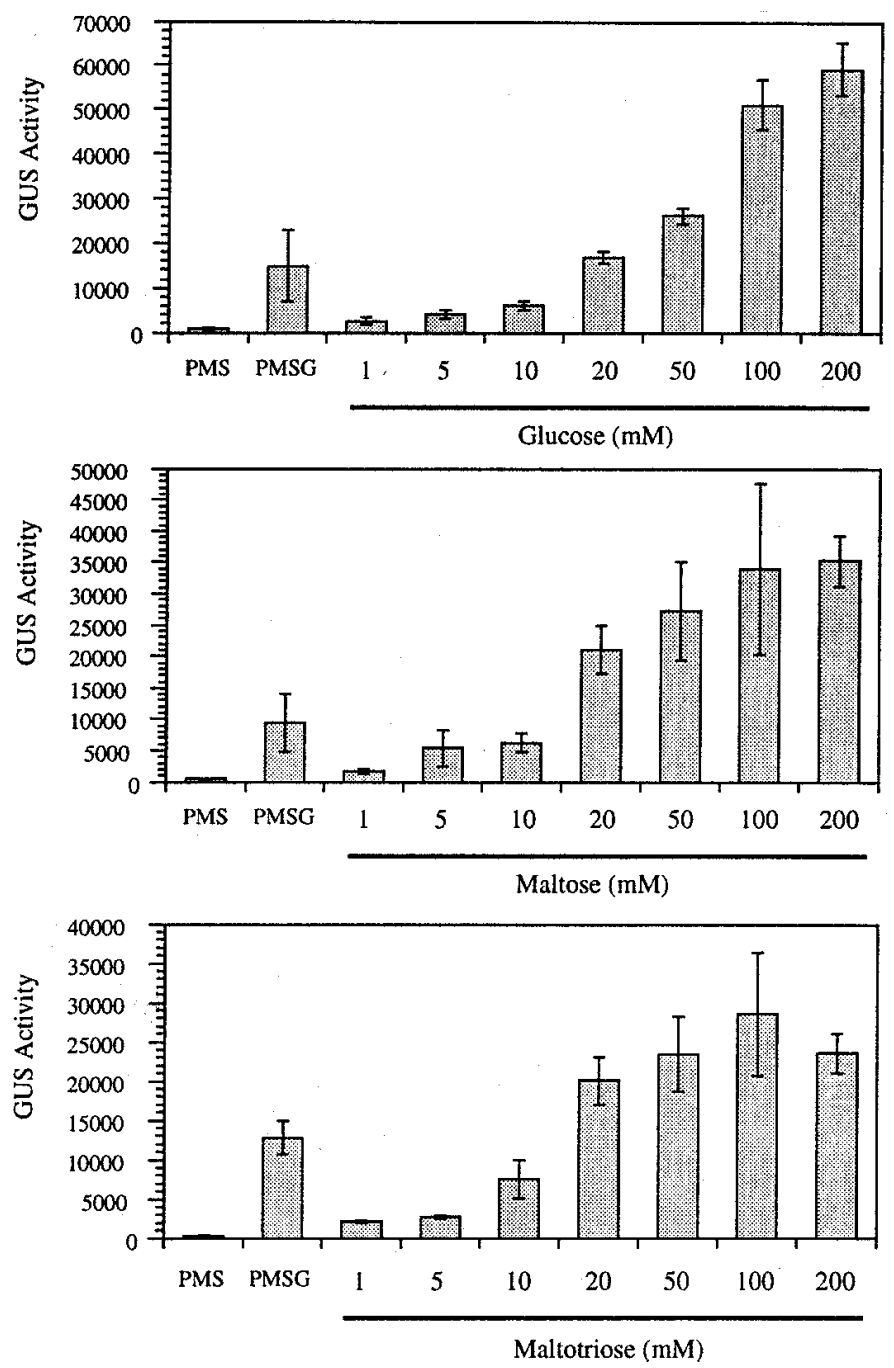

Fig. 6. Effect of various concentrations of glucose, maltose, and maltotriose on the accumulation of $\beta$-glucuronidase (GUS) activity in transformant GAP13-22. Cultures were grown in peptone mineral salts (PMS) medium for 3 days at $28^{\circ} \mathrm{C}$. At zero time the culture medium in the bioassay was replaced. GUS activity was determined after $24 \mathrm{~h}$ of incubation. Values are the average of three replicates and the bars represent the standard errors. GUS values are $\mu$ moles 4 -methylumbelliferyl $/ \mathrm{min} / \mathrm{mg}$ of protein. PMS and PMS plus $60 \mathrm{~g} /$ liter of glucose (PMSG) are the negative and positive controls, respectively. by TLC. Glucose and maltose were produced from maltotriose, maltotetraose, and maltoheptaose, but maltose was not hydrolyzed to glucose (Fig. 5).

Induction of GUS by various carbohydrates. Glucose, maltose, and maltotriose were tested in the GUS-expression bioassay and found to induce GUS activity at concentrations as low as 1 $\mathrm{mM}$ (Fig. 6). Increasing concentrations of glucose up to $200 \mathrm{mM}$ resulted in a corresponding increase in GUS activity. In contrast, the level of GUS activity induced by maltose and maltotriose reached a maximum between 20 and $200 \mathrm{mM}$. When these sugars were tested together in various combinations, no synergism was detected (data not shown). Deoxyglucose $(100 \mathrm{mM})$ was tested in the assay and did not induce GUS activity (data not shown). Sorbitol and fructose induced GUS activity to a similar level as glucose (Fig. 7). Lactose, sorbose, and glycerin were poor inducers (Fig. 7).

\section{DISCUSSION}

A goal of this research was to characterize the aflatoxin-inducing factor(s) detected in the culture filtrate of maize kernels colonized by A. flavus. The presence of glucose, maltose, and maltotriose in the culture filtrates suggests that these sugars are part of the aflatoxin-inducing activities. It has long been established that the source of carbohydrates available to A. flavus or A. parasiticus greatly influences aflatoxin production. Studies by Diener, Buchannan, and others showed that glucose, sucrose, and maltose support high levels of aflatoxin; whereas, carbon sources such as peptone, lactose, and xylose support growth of the fungi, but do not produce high levels of aflatoxin production $(1,2,7,13,23,27$, 34). Abdollahi and Buchanan (2) showed that the glucose analogs $\alpha$-methyl-D-glucose and 3-0-methyl-D-glucose did not induce aflatoxin. From these studies, it was hypothesized that the best inducers of aflatoxin biosynthesis were carbon sources readily metabolized by glycolysis and the pentose phosphate pathway. The molecular data obtained in the present study confirmed much of the work published by these earlier researchers and support their hypothesis. Carbohydrates such as glucose, maltose, maltotriose, fructose, and sorbitol resulted in the highest level of induction of GUS activity, whereas peptone, lactose, sorbose, and glycerin were poor inducers. The glucose analogue 2-deoxy-D-glucose also was tested and found not to induce GUS activity.

The minimum glucose concentration for the induction of aflatoxin biosynthesis also was determined by the earlier researchers. Wiseman and Buchanan (34) reported the induction of aflatoxin biosynthesis by $A$. parasiticus $48 \mathrm{~h}$ after replacing the PMS medium with $100 \mathrm{mM}$ glucose. Lower concentrations (1 and $10 \mathrm{mM})$ of glucose failed to induce detectable aflatoxin production. Using

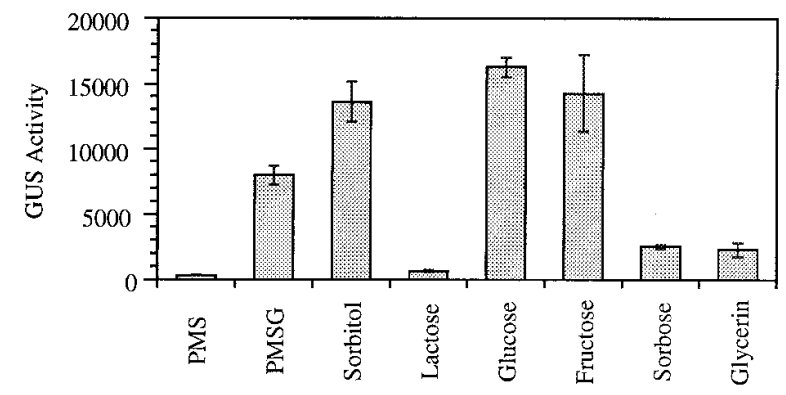

Fig. 7. Effect of various carbon sources on the accumulation of $\beta$-glucuronidase (GUS) activity in transformant GAP13-22. Cultures were grown in peptone mineral salts (PMS) medium for 3 days at $28^{\circ} \mathrm{C}$. At zero time the culture medium in the bioassay was replaced with $100 \mathrm{mM}$ solution of the various sugars. GUS activity was determined after $24 \mathrm{~h}$ of incubation. Values are the average of three replicates and the bars represent the standard errors. GUS values are $\mu$ moles 4-methylumbelliferyl $/ \mathrm{min} / \mathrm{mg}$ of protein. PMS and PMS plus $60 \mathrm{~g} /$ liter of glucose (PMSG) are the negative and positive controls, respectively. 
the GUS-reporter assay, we have shown that a gene involved in aflatoxin biosynthesis was induced within $24 \mathrm{~h}$ after replacement of the medium with glucose concentrations of $1 \mathrm{mM}$. It was also shown that maltose and maltotriose induced GUS activity at similar concentrations. These data indicate that an assay measuring aflatoxin gene expression is more sensitive than measuring aflatoxin directly. The data also indicate that a mechanism regulating aflatoxin biosynthesis is activated by low levels of carbon and that increasing the carbon concentration accentuates the induction process.

The carbon sources that induce aflatoxin are most likely metabolized by alcoholic fermentation. This conclusion is based on several observations. Aflatoxin-producing cultures have been shown to have degenerated mitochondria (7), accumulate ethanol (29), and have increased expression of the alcohol dehydrogenase gene $a d h 1$ (35). Remaining unresolved is whether the induction of aflatoxin biosynthesis occurs in response to internal energy levels (1), by changes in the level of cyclic nucleotides (22), or by other factors.

Analysis of the culture filtrates suggests that a single $\alpha$-amylase is responsible for starch-degrading activity. No evidence was found to indicate that maltase or amyloglucosidase was produced to any significant level in the culture filtrates. These data are in agreement with Khoo et al. (19), who purified an $\alpha$-amylase from A. flavus grown on raw tapioca starch. A. flavus appears to be different from the closely related species $A$. oryzae, which produces both $\alpha$-amylase and amyloglucosidase when grown on starch (16).

Several reports indicate that $A$. flavus preferentially colonizes the embryo tissue upon infection of maize kernels $(5,6,18)$. All indications are that the endosperm is not significantly affected by the fungus until late in the destruction of the kernel. Aflatoxin is also produced predominantly in the embryo tissue $(5,6,18)$. A . flavus may colonize the embryo tissue first, because it is richer in nitrogen and other nutrients than the endosperm. Although starch is the primary component of the endosperm, the embryo also has significant amounts of starch, as much as $8 \%$ of the dry weight (32). Increase in amylase activity in the filtrates from maize kernel cultures paralleled the increase in aflatoxin-inducing activity, suggesting that the action of amylase on the maize starch has a role in induction. We hypothesize that, after embryo colonization, A. flavus produces an extracellular amylase that supplies a burst of fermentable sugars, and it is these sugars that induce aflatoxin biosynthesis. A similar pattern of amylase production was observed when $A$. flavus was grown on amylopectin as a carbon source; however, the filtrates from the $0.2 \%$ amylopectin cultures did not contain measurable amounts of glucose, maltose, or maltotriose and did not induce GUS activity. When the initial amylopectin was increased 10 -fold, inducing activity and these sugars were detected in the 2-day culture filtrates. These data suggest that the products of amylopectin degradation were limiting in the $0.2 \%$ culture. As a consequence, the products were likely rapidly consumed by the growing fungus and not available to stimulate aflatoxin biosynthesis.

Although the data presented are not conclusive that amylase has a role in the production of aflatoxin in infected maize, they suggest that inhibiting the action of the amylase produced by $A$. fla$v u s$ may be a viable control strategy. An obvious question is do the various plant amylase inhibitors have any effect on amylases from A. flavus? Published literature suggest that many of the known amylase inhibitors, including maize, have little or no activity toward fungal amylases $(3,20)$. There is one report indicating that the inhibitor from black bean has activity against fungal amyloglucosidase, but it was not inhibitory to $\alpha$-amylases (21). We have tested wheat (Triticum aestivum) amylase inhibitor (Sigma Chemical Co., St. Louis) and determined that the inhibitor did not inhibit the amylase from A. flavus, but inhibited porcine pancreatic $\alpha$-amylase by $90 \%$ (C. P. Woloshuk and J. R. Cavaletto, unpublished data).

\section{ACKNOWLEDGMENTS}

Support for this research was provided by USDA cooperative agreement 58-6435-4-116. This report constitutes journal publication 15090 of the Purdue University Agricultural Experiment Station. We thank N. Carpita for sharing his expertise in carbohydrate analysis and H. Holen for his assistance with the isoelectric-focusing gels.

\section{LITERATURE CITED}

1. Abdollahi, A., and Buchanan, R. L. 1981. Regulation of aflatoxin biosynthesis: Characterization of glucose as an apparent inducer of aflatoxin production. J. Food Sci. 46:143-146.

2. Abdollahi, A., and Buchanan, R. L. 1981. Regulation of aflatoxin biosynthesis: Induction of aflatoxin production by various carbohydrates. J. Food Sci. 46:633-635.

3. Blanco-Labra, A., and Iturbe-Chinas, F. A. 1981. Purification and characterization of an $\alpha$-amylase inhibitor from maize (Zea maize). J. Food Biochem. 5:1-17.

4. Bradford, M. M. 1976. A rapid and sensitive method for the quantitation of microgram quantities of protein utilizing the principle of protein-dye binding. Anal. Biochem. 72:248-254.

5. Brown, R. L., Cleveland, T. E., Payne, G. A., Woloshuk, C. P., Campbell, K. W., and White, D. G. 1994. Determination of resistance to aflatoxin contamination in maize kernels and of fungal colonization using an A. flavus transformant expressing Escherichia coli $\beta$-glucuronidase. Phytopathology 85:983-989.

6. Brown, R. L., Cotty, P. J., Cleveland, T. E., and Widstom, N. W. 1993. Living maize embryo influences accumulation of aflatoxin in maize kernels. J. Food Prot. 56:967-971.

7. Buchanan, R. L., Jones, S. B., Gerasimowicz, W. V., Zaika, L. L., Stahl, H. G., and Ocker, L. A. 1987. Regulation of aflatoxin biosynthesis: Assessment of the role of cellular energy status as a regulator of the induction of aflatoxin production. Appl. Environ. Microbiol. 53:1224-1231.

8. Buchanan, R. L., and Stahl, H. G. 1984. Ability of various carbon sources to induce and support aflatoxin synthesis by Aspergillus parasiticus. J. Food Saf. 6:271-279.

9. Campbell, K. W., and White, D. G. 1994. An inoculation devise to evaluate maize for resistance to ear rot and aflatoxin production by $A s$ pergillus flavus. Plant Dis. 78:778-781.

10. Campbell, K. W., White, D. G., Toman, J., and Rocheford, T. R. 1993. Source of resistance in $\mathrm{F}_{1}$ corn hybrids to ear rot caused by Aspergillus flavus. Plant Dis. 77:1169.

11. Chang, P.-K., Skory, C. D., and Linz, J. E. 1992. Cloning of a gene associated with aflatoxin $\beta 1$ biosynthesis in Aspergillus parasiticus. Curr. Genet. 21:231-233.

12. Cleveland, T. E., and McCormick, S. P. 1987. Identification of pectinases produced in cotton bolls infected with Aspergillus flavus. Phytopathology 77:1498-1503.

13. Davis, N. D., and Diener, U. L. 1968. Growth and aflatoxin production by Aspergillus parasiticus from various carbon sources. Appl. Microbiol. 16:158-159.

14. Diener, U. F., Cole, R. J., Sanders, T. H., Payne, G. A., Lee, L. S., and Klich, M. A. 1987. Epidemiology of aflatoxin formation by Aspergillus flavus. Annu. Rev. Phytopathol. 25:249-270.

15. Flaherty, J. E., Weaver, M. A., Payne, G. A., and Woloshuk, C. P. 1995. A $\beta$-glucuronidase reporter gene construct for monitoring aflatoxin biosynthesis in Aspergillus flavus. Appl. Environ. Microbiol. 61:2482-2486.

16. Fogarty, W. M. 1994. Enzymes of the genus Aspergillus. Pages 177-218 in: Aspergillus. J. E. Smith, ed. Plenum Press, New York.

17. Gorman, D. P., and Kang, M. S. 1987. Preharvest aflatoxin contamination in maize: Resistance and genetics. Plant Breeding 107:1-10.

18. Keller, N. P., Butchko, R. A. E., Sarr, B., and Phillips, T. D. 1994. A visual pattern of mycotoxin production in maize kernels by Aspergillus spp. Phytopathology 84:483-488.

19. Khoo, S. L., Amirul, A.-A., Kamaruzaman, M., Nazalan, N., and Asisan, M. N. 1994. Purification and characterization of $\alpha$-amylase from Aspergillus flavus. Folia Microbiol. 39:392-398.

20. Kutty, A. V. M., and Pattabiraman, T. N. 1986. Isolation and characterization of an amylase inhibitor from sorghum seeds, specific for human enzymes. J. Agric. Food Chem. 34:552-567.

21. Lajolo, F. M., and Filho, F. F. 1985. Partial characterization of the amylase inhibitor of black beans (Phaseolis vulgaris), variety Rico 23. J. Agric. Food Chem. 33:132-138.

22. Luchese, R. H., and Harrigan, W. F. 1993. Biosynthesis of aflatoxin: The role of nutritional factors. J. Appl. Bacteriol. 74:5-14.

23. Mateles, R. I., and Adye, J. C. 1965. Production of aflatoxins in submerged culture. Appl. Microbiol. 13:208-211.

24. Nelson, M. 1944. A photometric adaptation of the Somoqui method for 
the determination of glucose. J. Biol. Chem. 153:375-380.

25. Payne, G. A. 1992. Aflatoxin in maize. Crit. Rev. Plant Sci. 10:423-440.

26. Payne, G. A., Nystrom, G. J., Bhatnagar, D., Cleveland, T. E., and Woloshuk, C. P. 1993. Cloning of the afl-2 gene involved in aflatoxin biosynthesis from Aspergillus flavus. Appl. Environ. Microbiol. 59:156162.

27. Reddy, T. V., Viswanathan, L., and Venkitasubramanian, T. A. 1979. Factors affecting aflatoxin production by Aspergillus parasiticus in a chemically defined medium. J. Gen. Microbiol. 114:409-413.

28. Reid, J. L., and Collmer, A. 1985. Activity stain for rapid characterization of pectic enzymes in isoelectric focusing and sodium dodecylsulfate-polyacrylamide gels. Appl. Environ. Microbiol. 50:615-622.

29. Sanchis, V., Vinas, I., Robert, I. N., Jeenes, D. J., Watson, A. J., and Archer, D. B. 1994. A pyruvate decarboxylase gene from Aspergillus parasiticus. FEMS (Fed. Eur. Microbiol. Soc.) Microbiol. Lett. 117:207210.

30. Scott, G. E., and Zummo, N. 1992. Registration of Mp420 germplasm line of maize. Crop Sci. 32:1296.

31. Skory, C. D., Chang, P.-K., Cary, J., and Linz, J. E. 1992. Isolation and characterization of a gene from Aspergillus parasiticus associated with the conversion of versicolorin A to sterigmatocystin in aflatoxin biosynthesis. Appl. Environ. Microbiol. 58:3527-3537.

32. Watson, S. A. 1987. Structure and composition. Pages 53-82 in: Corn: Chemistry and Technology. S. A. Watson and P. E. Ramstad, eds. American Association of Cereal Chemists, St. Paul, MN.

33. Widstrom, N. W., McMillian, W. W., and Wilson, D. 1987. Segregation for resistance to aflatoxin contamination among seeds on an ear of hybrid maize. Crop Sci. 27:961-963.

34. Wiseman, D. W., and Buchanan, R. L. 1987. Determination of glucose level needed to induce aflatoxin production in Aspergillus parasiticus. Can. J. Microbiol. 33:828-830.

35. Woloshuk, C. P., and Payne, G. A. 1994. The alcohol dehydrogenase adh1 is induced in Aspergillus flavus grown on medium conducive to aflatoxin biosynthesis. Appl. Environ. Microbiol. 60:670-676.

36. Woloshuk, C. P., Payne, G. A., Foutz, K. R., Brewer, J. F., Bhatnagar, D., and Cleveland, T. E. 1994. Molecular characterization of aflR, a regulatory gene for aflatoxin biosynthesis. Appl. Environ. Microbiol. 60:24082414. 